\title{
A Fuzzy Probabilistic Maximum Technique to Optimize an Unconstrained Utility Based Multi Objective Model
}

\author{
Mohammadreza Torkjazi ${ }^{1}$ and Hamed Fazlollahtabar ${ }^{2 *}$
}

${ }^{1}$ Department of Industrial Engineering, Mazandaran University of Science and Technology, Babol, Iran

${ }^{2}$ Faculty of Industrial Engineering, Iran University of Science and Technology, Tehran, Iran

\begin{abstract}
One way to optimize multi objective mathematical models is to employ utility functions for the objectives. Recent studies on utility based multi objective optimization consider only one utility function for each objective. But, in reality it is not reasonable to have a unique utility function corresponding to each objective function. Here, an unconstrained multi objective mathematical model is considered in which several utility functions are associated for each objective. A fuzzy probabilistic approach is incorporated to investigate the uncertainty of the utility functions for each objective. Since these utility functions are uncertain and in fuzzy form, the total utility function of the problem is a fuzzy nonlinear mathematical model. While, there are not any conventional approaches to solve such a model, a defuzzification method to change the total utility function to a crisp nonlinear model is employed. Meanwhile, maximum technique is applied to defuzzify the conditional utility functions. Then, an existing method to optimize the final single objective nonlinear model is adapted. The obtained results show that by changing the utility functions regarding to the dynamism of the environment, the method is still capable to provide the solutions accordingly. The effectiveness of the proposed approach is shown by solving a test problem.
\end{abstract}

Keywords: Multi objective optimization; Fuzzy probabilistic; Utility function

\section{Introduction}

There are many real-life problems that are formulated as multiobjective optimization problems. The field of multi-objective optimization is attracting more and more attention, notably because it offers new opportunities for defining problems. The objectives may not always be limited to cost. In fact, numerous other aspects, such as balancing of workloads (time, distance, ...) can be taken into account simply by adding new objectives [1].

Multiple objectives often conflict with each other and require multi-objective approaches rather than a single objective approach [2]. There are three approaches to solve a multi-objective problem: Priori approaches, Interactive approaches, and A posteriori approaches [3]. The main question in this study is how multiple objectives having several utility functions can be optimized. So, a multiple objective mathematical model having several utility functions is optimized under uncertainty. Fuzzy probabilistic programming is developed for multiple utility functions.

The organization of this paper is as follows. Next, a review of the related literature is presented. In Section 3, the utility function method accompanying with our proposed method are described. In Section 4 a numerical example of a multi-objective problem is solved by the proposed method. Finally in Section 5 our conclusions are given.

\section{Literature Review}

From a decision maker perspective, the choice of a solution from all presented efficient solutions is called a posteriori approach. In the priori approach, the decision maker expresses his preference relative to the objectives in one of two ways. The first one consists of attaching weights to each objective and combining them in a linear function [46]. In the second approach, objectives are ranked in decreasing order of importance; the problem is solved for the first objective, and then the second problem is solved for the second objective under the constraint that the optimal solution of the first objective does not change. This single-objective problem process is continued until the last objective [7-11]. In the interactive approach, the decision maker intervenes in the optimization process to guide it to most suitable solutions $[12,13]$. In this paper we use the Utility function method to solve a multi-objective problem which is one of the methods of the priori approaches.

Over the second half of the $20^{\text {th }}$ century, optimization found widespread applications in the study of physical and chemical systems, production planning and scheduling systems, location and transportation problems, resource allocation in financial systems, and engineering design. From the very beginning of the application of optimization to these problems, it was recognized that analysts of natural and technological systems are almost always confronted with uncertainty. Approaches to optimization under uncertainty have followed a variety of modeling philosophies, including expectation minimization, minimization of deviations from goals, minimization of maximum costs, and optimization over soft constraints. A key difficulty in optimization under uncertainty is in dealing with an uncertainty space that is huge and frequently leads to very large-scale optimization models [14].

Research that considers uncertainty can be categorized according to the four primary approaches [14]:

(1) Stochastic programming approach,

(2) Fuzzy programming approach,

*Corresponding author: Hamed Fazlollahtabar, Faculty of Industrial Engineering Iran University of Science and Technology, Tehran, Iran, Tel: +98 217724054 ; E-mail: hfazl@iust.ac.ir

Received November 14, 2014; Accepted December 22, 2014; Published January 15,2015

Citation: Torkjazi M, Fazlollahtabar H (2015) A Fuzzy Probabilistic Maximum Technique to Optimize an Unconstrained Utility Based Multi Objective Model. Ind Eng Manage 3: 147. doi: 10.4172/2169-0316.1000147

Copyright: (c) 2015 Torkjazi M, et al. This is an open-access article distributed under the terms of the Creative Commons Attribution License, which permits unrestricted use, distribution, and reproduction in any medium, provided the original author and source are credited. 
(3) Stochastic dynamic programming approach, and

(4) Robust optimization approach.

In the first approach, some parameters are regarded as random variables with known probability distributions. The second one seeks the solution considering some variables as fuzzy numbers. The third one includes applications of random variables in dynamic programming which can be found essentially in all areas of multi-stage decision making. In comparison, the last one represents uncertainty through setting up different scenarios which demonstrate realizations of uncertain parameters. The aim of this approach is to find a robust solution which ensures that all specified scenarios are "close" to the optimum in response to changing input data [15].

However, much of decision making in the real-world takes place in an environment where the objectives, constraints or parameters are not known precisely [16]. Therefore a decision is often made on the basis of vague information or uncertain data. In 1970, Zadeh and Bellman introduced the fuzzy set theory into the traditional decision making problem involving uncertainty and imprecision. According to the fuzzy theory, the inaccurate objectives and constraints, called fuzzy objectives and constraints, are represented by associated membership functions [17]. In 1974, Tanaka initially proposed the concept of fuzzy mathematical programming, and in 1978, Zimmermann formulated fuzzy linear programming with several objectives [18-20].

Modeling real world problems typically involves processing uncertainty of two distinct types. These are uncertainty arising from a lack of knowledge relating to concepts which, in the sense of classical logic, may be well defined and uncertainty due to inherent vagueness in concepts themselves. Traditionally, the above are modeled in terms of probability theory and fuzzy set theory respectively. Furthermore, there are many situations where we have insufficient information regarding vague or fuzzy concepts. That is where both types of uncertainties are present. This suggests the need for a theory of the probability of fuzzy events [21].

\section{Proposed Method}

The Utility Function Method proposed by Keeney and Raiffa in 1976, is one of the approaches in solving a Multi-objective problem [22]. In this method, a conditional utility function should be assigned to each objective. For each objective, while the other objectives are fixed in their levels, we can check whether the objective is utility independent from other ones or not. Then the conditional utility functions should be combined and form the total utility function. The last step in their method is optimizing the total utility function which means maximizing the utility of the decision maker.

But there is a problem for using this method in real world, because this method does not assume all of the situations in the problem's environment. To simply explain these situations, consider a manufacturing environment in a company. Maximizing the profit is one of the most important goals to company managers. In this case, just one utility function for the given objective can be used which is maximizing the profit for an 8 hour shift. But sometimes there are also other shifts starting after the main shift for employees. Usually because of some factors such as employees' fatigue and environmental conditions or sometimes company's conditions, working in the main shift is more favorable to employees than working in the overtime shifts. Thus the same utility function cannot be used for the overtime period, while the objective function is maximizing the profit; another utility function for the overtime period has to be defined. So for better modeling of the problem in the real world and to have the nearest solutions to the ideal solution, different utility functions have to be defined for each objective based on different situations and different environments.

In this paper we assume that each objective function has several utility functions, while researchers in the past used to consider just one utility function for each objective.

Planning for a short time or a long time period is one of the manager's methods to control their company and compete with other companies as well as they can. Since these plans are for the future periods of time they can't use the crisp data in their planning. In other words, these plans are always considered under uncertainty. Assume that a plan is designed for a long time period of time for a company. To do this, different utility functions can be assigned to each one of the objectives based on the different situations in the company's environment. Because of the uncertainty conditions in the problem, each utility function corresponds to a fuzzy set and there is a probability for each utility function for occurring in the assumed period of the planning time. These functions can be called as Partial Utility Functions. So the first step in our method is calculating the probabilities of the partial utility functions. After that these partial utility functions should be combined to achieve the conditional utility function for each objective. So there will be only one conditional utility function for each objective. The next Step is combining conditional utility functions and creating the total utility function. At last by optimizing the total utility function, the utility of the decision maker can be maximized.

\section{Probability of a fuzzy event}

To calculate the probability of a function, the probability of all of the variables participating in the function should be used. In other words, a function will occur when all of the variables occur. So to achieve the probability of the function $u$, the probability of a set like $M$ which includes all of the variables of $u$ should be calculated. In this way, the probability of $M$ equals to the probability of function $u$.

For example consider the function below:

$u=a x_{1}+b x_{2}+c x_{3}+h$

and the probabilities of the variables are:

$$
\begin{aligned}
& P_{x_{1}}=p_{1}, P_{x_{2}}=p_{2}, P_{x_{3}}=p_{3} \\
& M=\left\{x_{1}, x_{2}, x_{3}\right\}
\end{aligned}
$$

Because the variables in this function are crisp variables, the probability of the set $M$ and the function $u$ is:

$$
P(u)=P(M)=p_{1}+p_{2}+p_{3}
$$

In this paper, the utility functions are in fuzzy environment and each of them is dependent to a fuzzy set which describes the uncertainty in the problem. So the crisp results cannot be achieved for their probabilities. To calculate the probability of these fuzzy functions, the Yager's method can be used [23]. The first step for calculating these probabilities is defining the set $M$ for each partial utility function. To do that, for every partial utility function, each variable should be selecte $\mathrm{d}$ from the crisp universe $X$ with a degree of membership based on the conditions of the occurrence of the function. Then these variables and their degree of membership can be used to create the set $M$ for the function.

Probability of a Fuzzy Event as a Fuzzy set (Yager's Method [24]): Let us assume that there exists a probability measure $\mathrm{P}$ defined on the 
Citation: Torkjazi M, Fazlollahtabar H (2015) A Fuzzy Probabilistic Maximum Technique to Optimize an Unconstrained Utility Based Multi Objective Model. Ind Eng Manage 4: 147. doi: 10.4172/2169-0316.1000147

Page 3 of 7

set of all crisp subsets of (the universe) $X$, the Borel set. $P\left(x_{i}\right)$ shall denote the probability of element $x_{i} \in X$. Let $\tilde{A}=\left\{\left(x, \mu_{A}(x) \mid x \in X\right\}\right.$ be a fuzzy set representing a fuzzy event. The degree of membership of element $x_{i} \in \tilde{A}$ is denoted by $\mu_{\tilde{A}}\left(x_{i}\right)$. $\alpha$-level sets or $\alpha$-cuts shall be denoted by $A_{\alpha}$.

In 1984, Yager [23] suggested that it is quite natural to define the probability of an $\alpha$-level set as

$$
P\left(A_{\alpha}\right)=\sum_{x \in A_{\alpha}} P(x)
$$

\section{Definition 1}

Let $A_{\alpha}$ be the a-level set of a fuzzy set $\tilde{A}$ representing a fuzzy event. Then the probability of a fuzzy event $\tilde{A}$ can be defined as

$$
P_{y}(\tilde{A})=\left\{\left(P\left(A_{\alpha}\right), \alpha\right) \mid \alpha \in[0,1]\right\}
$$

With the interpretation "the probability of at least an a degree of satisfaction to the condition $\tilde{A}$."

It is very clear that Yager [23] considers $\alpha$, which is used as the degree of membership of the probabilities $P\left(A_{\alpha}\right)$ in the fuzzy set $P_{y}(\tilde{A})$, as a kind of significance level for the probability of a fuzzy event.

Yager [23] also suggests another definition for the probability of a fuzzy event, which is derived as follows.

\section{Definition 2}

The truth of the proposition "the probability $\tilde{A}$ is at least w" is defined as the fuzzy set $P_{y}^{*}(\widetilde{A})$ with the membership function

$$
P_{y}^{*}(\tilde{A})(w)=\sup \left\{\alpha \mid P\left(A_{\alpha}\right) \geq w\right\}, w \in[0,1]
$$

It should be realized that now the "indicator" of significance of the probability measure is $\mathrm{w}$ and no longer $\alpha$.

On the other hand if we denote the complement of $\widetilde{A}$ by $C \tilde{A}=\left\{\left(x, 1-\mu_{\tilde{A}}(x)\right) \mid x \in X\right\}$ and the $\alpha$-level sets of $C \tilde{A}$ by $(C \tilde{A})$, then

$$
P_{y}^{*}(C \tilde{A})(w)=\sup \left\{\alpha \mid P\left(C A_{\alpha}\right) \geq w\right\}
$$

and $w \in[0,1]$ can be interpreted as the truth of the proposition "the probability of not $A$ is at least w."

Let us define $\bar{P}_{y}^{*}(\tilde{A})=1-P_{y}^{*}(C \tilde{A})$. If $\bar{P}_{y}^{*}(\tilde{A})(w)$ is interpreted as the truth of the proposition "probability of $\widetilde{A}$ is at most w", then we can argue as follows: The "and" combination of "the probability of $\widetilde{A}$ is at least w" and "the probability of $\widetilde{A}$ is at most w" might be considered as "the probability of $\tilde{A}$ is exactly w". If $P_{y}^{*}(\tilde{A})$ and $\bar{P}_{y}^{*}(\tilde{A})$ are considered as possibility distributions, then their conjunction is their intersection (modeled by applying the min-operator to the respective membership functions). Hence the following definition [24]:

\section{Definition 3}

Let $P_{y}^{*}(\widetilde{A})$ and $\bar{P}_{y}^{*}(\tilde{A})$ be defined as above. The possibility distribution associated with the proposition "the probability of $\widetilde{A}$ is exactly $w$ " can be defined as

$$
\bar{P}_{y}(\tilde{A})(w)=\min \left\{P_{y}^{*}(\tilde{A})(w), \bar{P}_{y}^{*}(\tilde{A})(w)\right\}
$$

For example consider the following fuzzy set: (Tables 1 and 2)

$$
\begin{aligned}
& \tilde{A}=\left\{\left(x_{1}, 1\right),\left(x_{2}, 0.7\right),\left(x_{3}, 0.6\right),\left(x_{4}, 0.2\right)\right\} \\
& P_{x_{1}}=0.1, P_{x_{2}}=0.4, P_{x_{3}}=0.3, P_{x_{4}}=0.2
\end{aligned}
$$

\begin{tabular}{|c|c|c|c|c|}
\hline$\alpha$ & $A_{\alpha}$ & $P(A)_{\alpha}$ & $w$ & $P_{y}^{*}(\tilde{A})(w)=\sup \alpha$ \\
\hline$[0,0.2]$ & {$\left[x_{1}, x_{2}, x_{3}, x_{4}\right]$} & 1 & {$[0.8,1]$} & 0.2 \\
\hline$[0.2,0.6]$ & {$\left[x_{1}, x_{2}, x_{3}\right]$} & 0.8 & {$[0.5,0.8]$} & 0.6 \\
\hline$[0.6,0.7]$ & {$\left[x_{1}, x_{2}\right]$} & 0.5 & {$[0.1,0.5]$} & 0.7 \\
\hline$[0.7,1]$ & {$\left[x_{1}\right]$} & 0.1 & {$[0,0.1]$} & 1 \\
\hline
\end{tabular}

Table 1: Fuzzy set 1.

\begin{tabular}{|c|c|c|c|c|c|}
\hline$\alpha$ & $(C A)_{\alpha}$ & $P(C A)_{\alpha}$ & $w$ & $P_{y}^{*}(C \tilde{A})(w)$ & $\bar{P}_{y}^{*}(\tilde{A})(w)=1-P_{y}(C \tilde{A})(w)$ \\
\hline 0 & {$\left[x_{1}, x_{2}, x_{3}, x_{4}\right]$} & 1 & {$[0.9,1]$} & 0 & 1 \\
\hline$[0,0.3]$ & {$\left[x_{2}, x_{3}, x_{4}\right]$} & 0.9 & {$[0.5,0.9]$} & 0.3 & 0.7 \\
\hline$[0.3,0.4]$ & {$\left[x_{3}, x_{4}\right]$} & 0.5 & {$[0.2,0.5]$} & 0.4 & 0.6 \\
\hline$[0.4,0.8]$ & {$\left[x_{4}\right]$} & 0.2 & {$[0,0.2]$} & 0.8 & 0.2 \\
\hline$[0.8,1]$ & 0 & 0 & 0 & 1 & 0 \\
\hline
\end{tabular}

Table 2: Fuzzy set 2

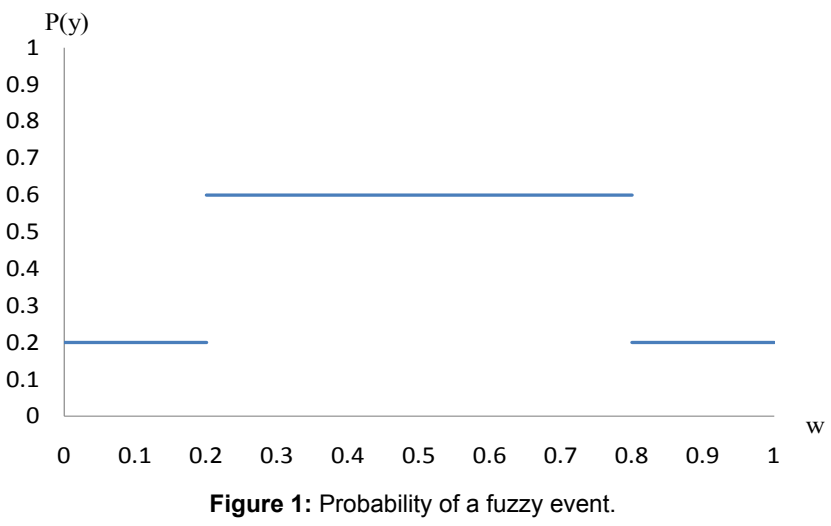

The probability $\bar{P}_{y}(\tilde{A})$ of the fuzzy event $\tilde{A}$ is now determined by the intersection of the fuzzy sets $P_{v}^{*}(\tilde{A})$ and $\bar{P}(A)$ modeled by the min-operator as in definition 3 (Figure 1):

$$
\bar{P}_{y}(\tilde{A})(w)=\left\{\begin{array}{ll}
0, & w=0 \\
0.2, & w \in[0,0.2] \\
0.6, & w \in[0.2,0.8] \\
0.2, & w \in[0.8,1]
\end{array}\right\}
$$

For example consider a problem with two objectives in which each objective corresponds to three partial utility functions.

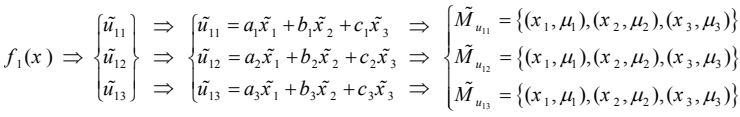

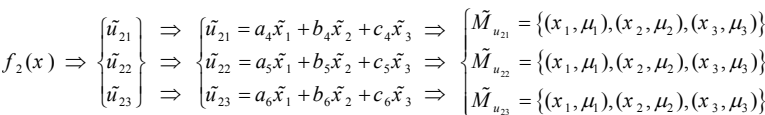

Now, the probabilities of the partial utility functions can be computed by using the Yager's method [23]. 
Citation: Torkjazi M, Fazlollahtabar H (2015) A Fuzzy Probabilistic Maximum Technique to Optimize an Unconstrained Utility Based Multi Objective Model. Ind Eng Manage 4: 147. doi: 10.4172/2169-0316.1000147

\section{Combining the partial utility functions}

The next step for solving the problem is combining the partial utility functions and achieving the conditional utility function for each objective. Consider the objective function (5) shown above. To do this, a coefficient for each partial utility function is needed. To achieve those coefficients we can use the probabilities calculated in the section 5.1.1.

On the other hand, as it's been shown, the probabilities of the partial utility functions are fuzzy sets and each member of these sets is a probability period with a degree of membership, while a crisp coefficient for each utility function is needed. So, to deal with this, some levels of probabilities can be used by cutting these fuzzy sets of probabilities based on the decision maker's opinion. Since the crisp probabilities for each variable were available at first for the whole planning period so the probability of each partial utility function has been calculated for the whole planning period. But in the proposed method, it has been decided to divide the whole period to partial periods and then assign a partial utility function to each one of these partial periods. So it is rational to use some cuts on the fuzzy sets of probabilities.

For example consider a company with two shifts for its employees and the objective is maximizing the profit. After checking the conditions existing in the company, it's been decided that the objective should have two partial utility functions. On the other hand, the manager wants to achieve at least $60 \%$ of the company's profit in the first shift and if the profit of the first shift is less than $60 \%$ of the whole profit in the planning period, there will be no utility or satisfaction for the manager. So the partial utility function of the first shift should occur at least $60 \%$ of the whole planning period and in result, the partial utility function for the second shift should also occur at most $40 \%$ of the whole period. The manager's request should be applied on the probability sets and therefore those members of the probability set of the first partial utility function which are in the interval of $60 \%$ to $100 \%$ should be selected for this function and those members of the probability set of the second partial utility function which are in the interval $0 \%$ to $40 \%$ should be selected for the second utility function.

Now for calculating the coefficients, one can sum the degree of the memberships of the elements selected for each of the partial utility functions from the probability sets, call this summation " $s$ ". After that for each objective, we should sum all of these " $s$ " and then it will be called cumulative value " $S$ ". By dividing each " $s$ " by " $S$ ", a coefficient " $t$ " for each partial utility function will be achieved.

For example consider the following probabilities for two partial utility functions $\tilde{u}_{1}$ and $\tilde{u}_{2}$ :

$$
\begin{aligned}
& P_{u_{1}}=\{((0,0.3), 0.2),((0.3,0.6), 0.3),((0.6,0.8), 0.1),((0.8,1), 0.3)\} \\
& P_{u_{2}}=\{((0,0.2), 0.3),((0.2,0.4), 0.4),((0.4,0.7), 0.1),((0.7,1), 0.2)\}
\end{aligned}
$$

Consider that the manager requested that the function $\tilde{u}_{1}$ must occur at least $60 \%$ and the function $\tilde{u}_{2}$ must occur at most $40 \%$ of the whole period.

$$
\begin{aligned}
& s_{1}=\mu_{(0.6,0.8)}+\mu_{(0.8,1)}=0.1+0.3=0.4 \\
& s_{2}=\mu_{(0,0.2)}+\mu_{(0.2,0.4)}=0.3+0.4=0.7 \\
& S=s_{1}+s_{2}=0.3+0.7=1.1 \\
& t_{1}=\frac{s_{1}}{S}=0.36 \quad, \quad t_{2}=\frac{s_{2}}{S}=0.64
\end{aligned}
$$

As it's been shown, now, the coefficients of the partial utility functions can be calculated. Assume that after all of the calculations, the coefficients for the functions (5) and (6) are as

$$
\left.f_{1}(x) \Rightarrow\left\{\begin{array}{l}
\tilde{u}_{1} \\
\tilde{u}_{\mathrm{P}} \\
\tilde{u}_{\mathrm{B}}
\end{array}\right\} \Rightarrow\left\{\begin{array}{l}
t_{1} \\
t_{\mathrm{R}} \\
t_{\mathrm{B}}
\end{array}\right\} \quad f_{2}(x) \Rightarrow\left\{\begin{array}{l}
\tilde{u}_{2} \\
\tilde{u}_{2} \\
\tilde{u}_{3}
\end{array}\right\} \Rightarrow \begin{array}{l}
t_{2} \\
t_{2} \\
t_{3}
\end{array}\right\}
$$

Since the partial utility functions are not utility independent from each other, for combining them and creating the conditional utility functions, one can simply add them together with their coefficients.

$$
\begin{aligned}
& \tilde{U}_{1}=t_{11} \tilde{u}_{11}+t_{12} \tilde{u}_{12}+t_{13} \tilde{u}_{13} \\
& \tilde{U}_{2}=t_{21} \tilde{u}_{21}+t_{22} \tilde{u}_{22}+t_{23} \tilde{u}_{23}
\end{aligned}
$$

$\tilde{U}_{1}$ and $\tilde{U}_{2}$ are conditional utility functions for $f(x)$ and $f_{2}(x)$ respectively.

\section{Combining the conditional utility functions [22]}

Based on the Keeney and Raiffa [22], there are different forms to combine utility functions. In this paper, formation of The Multiplicative utility function is used. Let us first define mutual utility independence.

\section{Definition 4}

Attributes $X_{1}, X_{2}, \ldots, X_{n}$ are mutually utility independent if every subset of $\left\{X_{1}, X_{2}, \ldots, X_{n}\right\}$ is utility independent of its complement.

\section{Theorem 1}

If attributes $X_{1}, X_{2}, \ldots, X_{n}$ are mutually utility independent, then

$$
\begin{gathered}
u(x)=\sum_{i=1}^{n} k_{i} u_{i}(x)+K \sum_{\substack{i=1 \\
j>i}}^{n} k_{i} \cdot k_{j} \cdot u_{i}\left(x_{i}\right) \cdot u_{j}\left(x_{j}\right)+K^{2} \sum_{\substack{i=1 \\
j>i \\
i \succ j}}^{n} k_{i} \cdot k_{j} \cdot k_{\ell} \cdot u_{i}\left(x_{i}\right) \cdot u_{j}\left(x_{j}\right) \cdot u_{\ell}\left(x_{\ell}\right) \\
+\ldots+K^{n-1} k_{1} k_{2} \ldots k_{n} u_{1}\left(x_{1}\right) u_{2}\left(x_{2}\right) \ldots u_{n}\left(x_{n}\right)
\end{gathered}
$$

Where

1. $u$ is normalized by $u\left(x_{1}^{0}, x_{2}^{0}, \ldots, x_{n}^{0}\right)=0$ and $u\left(x_{1}^{*}, x_{2}^{*}, \ldots, x_{n}^{*}\right)=1$.

2. $u_{i}\left(x_{i}\right)$ is a conditional utility function on $X_{i}$ normalized by $u_{i}\left(x_{i}^{0}\right)=0$ and $u_{i}\left(x_{i}^{*}\right)=1, i=1,2, \ldots, n$.

3. $k_{i}=u\left(x_{i}^{*}, \bar{x}_{i}^{0}\right)$

4. $K$ is a scaling constant that is a solution to

$$
1+K=\prod_{i=1}^{n}\left(1+K \cdot k_{i}\right)
$$

\section{Defuzzification of the conditional utility functions}

As known, the conditional utility functions of the problem we considered are fuzzy functions. So after combining these functions by using the multiplicative formation, the Total utility function will be a non-linear fuzzy function that can't be solved with the existing methods. Therefore each conditional utility function should be defuzzified and be changed to a crisp function. There are many methods for defuzzification. One of these methods is using the Maximum technique.

Defuzzification of the conditional utility functions: Since the variables used in this problem are fuzzy numbers, we can use this technique to defuzzify each one of the variables and substitute them with fuzzy variables. This method gives the output with the highest membership function. This technique is given by algebraic expression as 


$$
\mu_{A}\left(x^{*}\right) \geq \mu_{A}(x) \quad \text { for all } x \in X
$$

where $\mathrm{x}^{*}$ is the defuzzified value. In other words, each one of the variables can be expressed as a fuzzy number as below:

$$
\tilde{x}_{1}=\left\{\left(x_{11}, \mu_{11}\right),\left(x_{12}, \mu_{12}\right),\left(x_{13}, \mu_{13}\right)\right\}
$$

Assume that

$$
\mu_{12} \succ \mu_{11} \succ \mu_{13} \text {, }
$$

So the deffuzified result will be

$$
x_{1}=x_{12} \text {. }
$$

$x_{1}$ is the difuzzified value of $\tilde{x}_{1}$ and from now on $\tilde{x}_{1}$ can be substituted with $x_{1}$ in the conditional utility function.

\section{Calculating the scaling constants}

After the defuzzification and achieving the crisp conditional utility functions, calculating the scaling constants to combine the conditional utility functions is needed. Based on the Eq. (9) which shows the formation of the Total utility function, each conditional utility function has a scaling constant. To achieve these constants the indifference level of the decision maker should be calculated. For example by asking the decision maker, his indifference level is concluded as

$$
\left(f_{1}, f_{2}\right) \approx(\beta, 0) \sim(0, \lambda) .
$$

Recall that $u\left(x_{i}\right)$ actually means $u\left(x_{1}^{0}, \ldots, x_{i-1}^{0}, x_{i}, x_{i+1}^{0}, \ldots, x_{n}^{0}\right)$. Hence we can define

$$
u\left(\mathrm{x}_{\mathrm{i}}\right)=k_{\mathrm{i}} u_{\mathrm{i}}\left(\mathrm{x}_{\mathrm{i}}\right) .
$$

So

$$
\begin{aligned}
& U\left(f_{1}, 0\right)=k_{1} U_{f_{1}}\left(f_{1}, 0\right), \\
& U\left(0, f_{2}\right)=k_{2} U_{f_{2}}\left(0, f_{2}\right) .
\end{aligned}
$$

So by considering the indifference level of the decision maker

$$
\begin{aligned}
& U(\beta, 0)=U(0, \lambda) \\
& k_{1} U_{f_{1}}(\beta, 0)=k_{2} U_{f_{2}}(0, \lambda) .
\end{aligned}
$$

In the Eq. (13), the conditional utility functions can be substituted with their values. So the unknown parameters will be just $k_{1}$ and $k_{2}$. In this way a relation between $k_{1}$ and $k_{2}$ can be achieved. Then, the fourth property of the theorem 1 to calculate all of the scaling constants can be used:

$$
1+K=\prod_{i=1}^{n}\left(1+K \cdot k_{i}\right)
$$

\section{Total utility function}

Calculating all of the constants and conditional utility functions and putting them in the Eq. (9) for combining them, results in the Total utility function " $U(F)$ " which is non-fuzzy and non-linear and the nonlinear programming methods can be used to solve this final model.

$$
U(F)=k_{1} U_{\left(f_{1}\right)}+k_{2} U_{\left(f_{2}\right)}+K \cdot k_{1} \cdot k_{2} \cdot U_{\left(f_{1}\right)} \cdot U_{\left(f_{2}\right)}
$$

\section{Numerical example}

A numerical application of the proposed method is presented in order to illustrate its capabilities in dealing with Multi-objective optimization problems.
Consider the following problem with two objectives:

$\min : f_{1}(x)=x_{1}^{2}+3 x_{1} x_{2}+4 x_{2}^{2}$

$\min : f_{2}(x)=2 x_{1}^{2}+x_{1} x_{2}+x_{2}^{2}$

In order to use the utility function method, at first each one of these objectives should be solved as a single-objective problem to find the optimum value of them. These optimum values will be considered as the values which their utility functions equals to 1 . In other words, if these objectives reach their optimum values, the utility level of the decision maker will be maximized which equals to 1 .

On the other hand, since the objectives of the problem are minimization, so an upper bound for these objectives should be defined. In this problem the value 10 as the upper bound is considered. So after solving each objective as a single-objective problem the following results will be achieved:

$$
\begin{gathered}
\min : f_{1}(x) \Rightarrow\left\{\begin{array} { l } 
{ f _ { 1 } ^ { * } = 0 . 0 0 4 6 2 } \\
{ x _ { 1 } ^ { * } = 0 . 0 4 3 } \\
{ x _ { 2 } ^ { * } = - 0 . 0 4 7 }
\end{array} \Rightarrow \left\{\begin{array}{l}
0.00462 \leq f_{1}^{*} \leq 10 \\
P_{x_{1}}=0.48 \\
P_{x_{2}}=0.52
\end{array},\right.\right. \\
\min : f_{2}(x) \Rightarrow\left\{\begin{array} { l } 
{ f _ { 2 } ^ { * } = 0 . 0 0 0 0 6 3 } \\
{ x _ { 1 } ^ { * } = 0 . 0 0 2 2 } \\
{ x _ { 2 } ^ { * } = 0 . 0 0 6 3 }
\end{array} \Rightarrow \left\{\begin{array}{l}
0.000063 \leq f_{2}^{*} \leq 10 \\
P_{x_{1}}=0.26 \\
P_{x_{2}}=0.74
\end{array}\right.\right.
\end{gathered}
$$

Based on the questions asked from the decision maker pertaining to the objectives of the problem, it has been decided to define two utility functions for each objective. The utility functions and their relating fuzzy sets are as follows:

$$
\begin{gathered}
f_{1}(x) \Rightarrow\left\{\begin{array}{l}
\tilde{u}_{11}^{\prime} \\
\tilde{u}_{12}^{\prime}
\end{array}\right\} \Rightarrow \begin{cases}\tilde{u}_{11}^{\prime}=\tilde{x}_{1}+3 \tilde{x}_{2} & \Rightarrow\left\{\begin{array}{l}
\tilde{M}_{11}=\left\{\left(x_{1}, 0.4\right),\left(x_{2}, 0.5\right)\right\} \\
\tilde{u}_{12}^{\prime}=2 \tilde{x}_{1}+2 \tilde{x}_{2}
\end{array} \tilde{M}_{12}=\left\{\left(x_{1}, 0.6\right),\left(x_{2}, 0.5\right)\right\},\right.\end{cases} \\
f_{2}(x) \Rightarrow\left\{\begin{array}{l}
\tilde{u}_{21}^{\prime} \\
\tilde{u}_{22}^{\prime}
\end{array}\right\} \Rightarrow \begin{cases}\tilde{u}_{21}^{\prime}=4 \tilde{x}_{1}+\tilde{x}_{2} & \Rightarrow\left\{\begin{array}{l}
\tilde{M}_{21}=\left\{\left(x_{1}, 0.7\right),\left(x_{2}, 0.8\right)\right\} \\
\tilde{u}_{22}^{\prime}=\tilde{x}_{1}+\tilde{x}_{2}
\end{array} \tilde{M}_{22}=\left\{\left(x_{1}, 0.3\right),\left(x_{2}, 0.2\right)\right\} .\right.\end{cases}
\end{gathered}
$$

Then the utility functions should be normalized:

$$
\begin{aligned}
& \overline{\tilde{u}_{1}}=\tilde{u}_{11}^{\prime}+\tilde{u}_{12}^{\prime}=3 \tilde{x}_{1}+5 \tilde{x}_{2} \text {, } \\
& \overline{\tilde{u}}_{2}=\tilde{u}_{21}^{\prime}+\tilde{u}_{22}^{\prime}=2 \tilde{x}_{1}+3 \tilde{x}_{2} \text {. } \\
& \left\{\begin{array}{l}
\tilde{u}_{11}=\frac{\tilde{u}_{11}^{\prime}}{\tilde{u}_{1}}=\frac{\tilde{x}_{1}+3 \tilde{x}_{2}}{3 \tilde{x}_{1}+5 \tilde{x}_{2}} \\
\tilde{u}_{12}=\frac{\tilde{u}_{12}^{\prime}}{\tilde{\tilde{u}}_{1}}=\frac{2 \tilde{x}_{1}+2 \tilde{x}_{2}}{3 \tilde{x}_{1}+5 \tilde{x}_{2}}
\end{array}, \quad\left\{\begin{array}{l}
\tilde{u}_{21}=\frac{\tilde{u}_{21}^{\prime}}{\tilde{\tilde{u}}_{2}}=\frac{4 \tilde{x}_{1}+\tilde{x}_{2}}{2 \tilde{x}_{1}+3 \tilde{x}_{2}} \\
\tilde{u}_{22}=\frac{\tilde{u}_{22}^{\prime}}{\tilde{\tilde{u}}_{2}}=\frac{\tilde{x}_{1}+\tilde{x}_{2}}{2 \tilde{x}_{1}+3 \tilde{x}_{2}}
\end{array}\right.\right.
\end{aligned}
$$

The probabilities of the partial utility functions presented in Table 1 obtained by performing the Yager's Method [23]:

After that the cuts over the probability sets should be applied based on the decision maker's opinion to calculate the coefficients as given in Tables 2-5.

$$
\begin{aligned}
& \tilde{U}_{\left(f_{1}\right)}=t_{11} \tilde{u}_{11}+t_{12} \tilde{u}_{12} \\
& \tilde{U}_{\left(f_{1}\right)}=0.64 \tilde{u}_{11}+0.36 \tilde{u}_{12}
\end{aligned}
$$




\begin{tabular}{|l|l|}
\hline$\tilde{u}$ & $\bar{P}_{y}(\tilde{M})$ \\
\hline$\tilde{u}_{11}$ & $\bar{P}_{y}\left(\tilde{M}_{11}\right)=\{(0,0),([0,0.48], 0.4),([0.48,0.52], 0.5),([0.52,1], 0.4)\}$ \\
\hline$\tilde{u}_{12}$ & $\bar{P}_{y}\left(\tilde{M}_{12}\right)=\{(0,0),([0,1], 0.5)\}$ \\
\hline$\tilde{u}_{21}$ & $\bar{P}_{y}\left(\tilde{M}_{21}\right)=\{(0,0),([0,0.26], 0.7),([0.26,0.74], 0.8),([0.74,1], 0.7)\}$ \\
\hline$\tilde{u}_{22}$ & $\bar{P}_{y}\left(\tilde{M}_{22}\right)=\{(0,0),([0,1], 0.2)\}$
\end{tabular}

Table 3: Probabilities of the partial utility functions.

\begin{tabular}{|c|c|c|c|c|}
\hline$\tilde{u}$ & $p$ & $s$ & $S_{1}$ & $t$ \\
\hline$\tilde{u}_{11}$ & {$[0.5,1]$} & $s_{11}=0.9$ & 0.9 & $t_{11}=0.64$ \\
\hline$\tilde{u}_{12}$ & {$[0,0.5]$} & $s_{12}=0.5$ & 1.4 & $t_{12}=0.36$ \\
\hline
\end{tabular}

Table 4: Coefficients of the partial utility functions.

\begin{tabular}{|c|c|c|c|c|}
\hline$\tilde{u}$ & $p$ & $s$ & $S_{2}$ & $t$ \\
\hline$\tilde{u}_{21}$ & {$[0.6,1]$} & $s_{21}=1.5$ & 1.5 & $t_{21}=0.88$ \\
\hline$\tilde{u}_{22}$ & {$[0,0.6]$} & $s_{22}=0.2$ & 1.7 & $t_{22}=0.12$ \\
\hline
\end{tabular}

Table 5: Coefficients of the partial utility functions.

$$
\begin{aligned}
& \tilde{U}_{\left(f_{1}\right)}=\frac{1.36 \tilde{x}_{1}+2.64 \tilde{x}_{2}}{3 \tilde{x}_{1}+5 \tilde{x}_{2}} \\
& \tilde{U}_{\left(f_{2}\right)}=t_{21} \tilde{u}_{21}+t_{22} \tilde{u}_{22} \\
& \tilde{U}_{\left(f_{2}\right)}=0.88 \tilde{u}_{21}+0.12 \tilde{u}_{22} \\
& \tilde{U}_{\left(f_{2}\right)}=\frac{3.64 \tilde{x}_{1}+\tilde{x}_{2}}{2 \tilde{x}_{1}+3 \tilde{x}_{2}}
\end{aligned}
$$

The next step in solving the problem with the proposed method is the Defuzzification of the utility functions. Since the maximum technique defuzzify the variables separately, so the defuzzified conditional utility functions are as

$$
\begin{aligned}
& \tilde{U}_{\left(f_{1}\right)}=\frac{1.36 \tilde{x}_{1}+2.64 \tilde{x}_{2}}{3 \tilde{x}_{1}+5 \tilde{x}_{2}} \Rightarrow U_{\left(f_{1}\right)}=\frac{1.36 x_{1}+2.64 x_{2}}{3 x_{1}+5 x_{2}} \\
& \tilde{U}_{\left(f_{2}\right)}=\frac{3.64 \tilde{x}_{1}+\tilde{x}_{2}}{2 \tilde{x}_{1}+3 \tilde{x}_{2}} \Rightarrow U_{\left(f_{2}\right)}=\frac{3.64 x_{1}+x_{2}}{2 x_{1}+3 x_{2}}
\end{aligned}
$$

After defuzzification of the utility functions the scaling constants should be calculated:

$$
\begin{aligned}
& \left\{\begin{array} { l } 
{ U _ { ( F ) } ( 1 0 , 1 0 ) = 0 } \\
{ U _ { ( F ) } ( 0 . 0 0 4 6 2 , 0 . 0 0 0 0 6 3 ) = 1 }
\end{array} \Rightarrow \left\{\begin{array}{l}
U_{(F)}(0.00462,0)=k_{1} \\
U_{(F)}(0,0.000063)=k_{2}
\end{array}\right.\right. \\
& \left\{\begin{array}{l}
U_{(F)}\left(f_{1}, 0\right)=k_{1} U_{\left(f_{1}\right)}\left(f_{1}, 0\right) \\
U_{(F)}\left(0, f_{2}\right)=k_{2} U_{\left(f_{2}\right)}\left(0, f_{2}\right) .
\end{array}\right.
\end{aligned}
$$

The indifference level of the decision maker has been described as $\left(f_{1}, f_{2}\right) \approx(1.5,0) \sim(0,0.000063)$

$U_{(F)}(1.5,0)=U_{(F)}(0,0.000063)$.

So, by using the Eq. (13)

$k_{1} \cdot U_{\left(f_{1}\right)}(1.5,0) \quad k_{2} \cdot U_{\left(f_{2}\right)}(0,0.000063)$

$k_{1} \cdot U_{()}(1.5,0) \quad k_{2}$.

On the other hand we know that

$$
\begin{aligned}
& \left\{\begin{array}{l}
U_{\left(f_{1}\right)}(10,10)=0 \\
U_{\left(f_{1}\right)}(0.00462,0)=1
\end{array}\right. \\
& \left\{\begin{array}{l}
U_{\left(f_{2}\right)}(10,10)=0 \\
U_{\left(f_{2}\right)}(0.000063,0)=1
\end{array}\right.
\end{aligned}
$$

and

$U_{\left(f_{1}\right)}(1.5,0)=0.85$

$k_{2}=0.85 k_{1}$.

So

$$
\begin{aligned}
& 1+K=\prod(1+K \cdot k) \\
& 1+K=\left(1+K \cdot k_{1}\right) \cdot\left(1+K \cdot k_{2}\right) \\
& K=\frac{1-k_{1}-k_{2}}{k_{1} k_{2}}=\frac{1-1.85 k_{1}}{0.85 k_{1}^{2}}
\end{aligned}
$$

In order to calculate the $k_{1}$ following procedure can be used. By asking from the decision maker we conclude that

$\{(0.00462,0.000063):(10,10)\} \sim(3,5)$

$$
\begin{aligned}
& \Rightarrow \frac{1}{2} \Rightarrow(0.00462,0.000063) \\
& \Rightarrow \frac{1}{2} \Rightarrow \quad(10,10)
\end{aligned}
$$

$U_{(F)}(3,5)=\frac{1}{2} U_{(F)}(0.00462,0.000063)+\frac{1}{2} U_{(F)}(10,10)=0.5$

So by considering this value in the Eq. (14)

$U(F)=0.5=k_{1} U_{\left(f_{1}=3\right)}+k_{2} U_{\left(f_{2}=5\right)}+K \cdot k_{1} \cdot k_{2} \cdot U_{\left(f_{1}=3\right)} \cdot U_{\left(f_{2}=5\right)}$.

On the other hand we know that

$U_{\left(f_{1}\right)}(3,0)=0.7$
$U_{\left(f_{2}\right)}(0,5)=0.5$

So by solving Eq. (15)

$k_{1}=0.32, \quad k_{2}=0.27, \quad K=4.69$.

At last the Total Utility Function will be expressed as

$U(F)=U\left(f_{1}, f_{2}\right)=0.32\left(U_{\left(f_{1}\right)}\right)+0.27\left(U_{\left(\sigma_{2}\right)}\right)+0.4\left(U_{\left(f_{1}\right)} U_{\left(f_{2}\right)}\right)$

$U\left(f_{1}, f_{2}\right)=\left(\frac{0.43 x_{1}+0.84 x_{2}}{3 x_{1}+5 x_{2}}\right)+\left(\frac{0.98 x_{1}+0.27 x_{2}}{2 x_{1}+3 x_{2}}\right)+\left(\frac{1.98 x_{1}^{2}+4.39 x_{1} x_{2}+1.01 x_{2}^{2}}{6 x_{1}^{2}+19 x_{1} x_{2}+15 x_{2}^{2}}\right)$

and the problem is changed in to the following form 
Citation: Torkjazi M, Fazlollahtabar H (2015) A Fuzzy Probabilistic Maximum Technique to Optimize an Unconstrained Utility Based Multi Objective Model. Ind Eng Manage 4: 147. doi: 10.4172/2169-0316.1000147

Page 7 of 7

$\max U\left(f_{1}, f_{2}\right)$.

Solving the nonlinear problem (16) results in the following optimum values for the objectives and the utility level of the decision maker

$$
\begin{aligned}
U^{*}\left(f_{1}, f_{2}\right) & =0.96 \\
x^{*} & =(2.4691,0) \\
F^{*} & =(6.096,12.193)
\end{aligned}
$$

Note that the numerical example is based on a production system and the related data are taken from the normal production shift. The utility functions are formed regarding to the management policies. The example can be designed with respect to other utilities or other objectives whether minimize or maximize, and the procedure can be repeated to obtain the solution. For example one can substitute this problem with a more complicated constrained multi objective problem which has so many factors to be considered in the process of the algorithm., i.e., the complexity does not influence the solution capability.

\section{Conclusion}

This paper proposed a new method to solve multi-objective problem using multiple utility functions for each objective. In the classic utility method there is only one utility function for each objective. Based on the different situations in the programming environment and the uncertainty in decision making, fuzzy consideration was included. To solve the problem, Yager's method in the field of fuzzy probabilistic and the combination formation of conditional utility functions suggested by Keeney and Raiffa [22,23] called multiplicative, were adapted. Since the conditional utility functions were in the fuzzy environment, the Maximum Technique was used to defuzzify the functions. Finally the total utility function of the problem was optimized to achieve the best solutions for the objectives and maximize the utility of the decision maker.

To check the capability of the proposed method, a numerical illustration extracted from an industrial unit has been applied in the form of an unconstrained multi-objective problem. Based on the experimental results it can be concluded that the proposed methodology enables the decision making process for optimizing an unconstrained multi-objective problem using the utility function method under uncertainty.

As future researches the following topics could be of interest: Here we considered linear utility functions while exponential utility functions could also be worked on, using linguistic variables as utility based on preferences of decision makers, surveying the fuzzy transition matrix among multiple utility functions.

\section{References}

1. Kubotani H,Yoshimura K (2003) Performance evaluation of acceptance probability functions for multi-objective SA. Computers and Operations Research 30: 427-442.

2. Yang L, Jones BF, Yang S (2007) A fuzzy multi-objective programming for optimization of fire station locations through genetic algorithms. European Journal of Operational Research 181: 903-915.

3. Jozefowiez N, Semet F, Talbi E (2008) Multi-objective vehicle routing problems. European Journal of Operational Research 189: 293-309.

4. Rajendran C (1995) Heuristics for scheduling in flowshop with multiple objectives. European Journal of Operational Research 82: 540-555.

5. Nagar A, Heragu S, Haddock Y (1995) A branch and bound approach for a 2-machine flowshop scheduling problem. Journal of the Operational Research Society 46: 721-734.

6. Serifoglu FS, Ulusoy G (1998) A bicriteria two-machine permutation flowshop problem. European Journal of Operational Research 107: 414-430.

7. Rajendran C (1992) Two-stage flow shop scheduling problem with bicriteria Journal of the Operational Research Society 43: 871-884.

8. Gupta JND, Palanimuthu N, Chen CL (1999) Designing a tabu search algorithm for the two-stage flow shop problem with secondary criterion. Production Planning and Control 10: 251-265.

9. Gupta JND, Neppalli VR, Werner F (2001) Minimizing total flow time in a twomachine flowshop problem with minimum makespan. International Journal of Production Economics 69: 323-338

10. Gupta JND, Hennig K, Werner F (2002) Local search heuristics for two-stage flow shop problems with secondary criterion. Computers and Operations Research 29: 123-149.

11. T'kindt V, Billaut JC (2001) Multicriteria scheduling problems: A survey. RAIRO Operations Research 35: 143-163.

12. Bernardo JJ, Lin KS (1994) An interactive procedure for bi-criteria production scheduling. Computers and Operations Research 21: 677-688.

13. Arroyo JEC, Armentano VA (2005) Genetic local search for multi-objective flowshop scheduling problems. European Journal of Operational Research 167: 717-738.

14. Sahinidis NV (2004) Optimization under uncertainty: state-of-the-art and opportunities. Computers and Chemical Engineering 28: 971-983.

15. Mirzapour Al-e-hashem SMJ, Malekly H, Aryanezhad MB(2011) A multiobjective robust optimization model for multi-product multi-site aggregate production planning in a supply chain under uncertainty. Int. J. Production Economics 134: 28-42.

16. Liu B (2002) Theory and Practice of Uncertain Programming. Physica-Verlag Heidelberg.

17. Bellman RE, Zadeh LA (1970) Decision-making in a fuzzy environment. Management Science 17: 141-164.

18. Tanaka H, Okuda T, Asai K (1973) On fuzzy mathematical programming Journal of Cybernetics 3:37-46.

19. Zimmermann HJ (1978) Fuzzy programming and linear programming with several objective functions. Fuzzy Sets and Systems1:45-55.

20. Li S, Hu C (2009) Satisfying optimization method based on goal programming for fuzzy multiple objective optimization problem. European Journal of Operational Research 197:675-684.

21. Baldwin JF, Lawry J, Martin TP (1996) A mass assignment theory of the probability of fuzzy events. Fuzzy Sets and Systems83:353-367.

22. Keeney RL, Raiffa H (1993) Decisions with Multiple Objectives. Cambridge University Press: 289-305.

23. Yager RR (1984) A representation of the probability of fuzzy subsets.Fuzzy Sets and Systems13:273-283.

24. Zimmermann HJ (1996) Fuzzy Set Theory and Its Application, Kluwer Academic Publishers Group: 109-121. 